\title{
Development of a fingerprint singularity detection method based on moment invariants for biometrics and medical applications
}

\author{
L.V Dang ${ }^{1}$, S.S Makhanov ${ }^{1}$, L.C Hieu², M.S Packianather ${ }^{3}$, H.L Minh ${ }^{4}$, L.H Quoc ${ }^{4}$ \\ ${ }^{1}$ Sirindhorn IIT, Thammasat University, Pathum Thani, Thailand \\ ${ }^{2}$ Faculty of Engineering and Science, University of Greenwich, Kent, United Kingdom \\ ${ }^{3}$ School of Engineering, Cardiff University, Cardiff, United Kingdom \\ ${ }^{4}$ Department of S\&T Management, Saigon Hi-Tech Park, Ho Chi Minh, Vietnam \\ haidang3a2009@gmail.com
}

\begin{abstract}
The biometric technologies have long been used for identification and authentication purposes; and fingerprint is one of the most widely used biometric technologies. In medicine and healthcare applications, biometric systems are used to identify patients and retrieve crucial medical records. In this paper, we propose a fingerprint singularity detection algorithm based on a very well-known pattern recognition technique. The successfully developed algorithm was tested for different fingerprint image resolutions, noise levels, and it was directly compared to the traditional technique, Poincare index which is the scalar values representing the geometrical behavior of basic patterns. The test of the proposed algorithm shows the outperformed results in both the high noise and low resolution images. Especially, the descriptors can be extracted directly from the suspect original and sample fingerprint images. The proposed method is therefore robust and can be adopted to any special descriptors rather than the pure core and delta points. With the recent advancement in data science, the successfully developed algorithm is potential for development of innovative biometric and medical applications, especially for telehealth and e-health systems.
\end{abstract}

Keywords: Biometrics, Poincare index, Singularity detection, Medical records, Patient identification.

\section{Introduction}

Patient's privacy and data are the first and foremost important issues in today's healthcare, and security of Electronic Health Records (EHRs) which are the most vulnerable and tempting targets to cyber-attacks. To access confidential EHRs, the security systems for human authentication are normally used, including iris, DNA, voice recognition, fingerprint, retina and finger vein $[1,2]$, in which the fingerprint is one of the most widely used and well researched [3]. The use of fingerprint authentication to safeguard privacy and grant access to EHRs has been successfully developed and applied in healthcare [1, 4]. Generally, the applications of biometrics such as fingerprint in healthcare include: (1) to fight against health care fraud and abuse; (2) to manage and 
protect confidential EHRs; (3) to identify patients, especially for cases in which unconscious individual could be identified, and for speeding up patient intake and improving record accuracy, as well as preventing the duplication of medical records and potential medical record errors; (4) to safely operate medical facilities and equipment; and (5) to have a continuous and secure access to EHRs, especially in the cloud-based telehealth and telemedicine. There is an emerging need to enhance the security, effective managements and use of patient data and medical records, especially in telehealth, management of confidential medical records, and e-health systems.

This study aims to investigate the use of fingerprints for biometrics and medical applications, in which the fingerprint singularity detection method is used to correctly locate the singular points (core and delta points) of a fingerprint image for the fingerprint recognitions, based on the moment invariant which is an excellent technique for pattern recognition and classification.

In fingerprint singularity detection, the global structure of the fingerprint image is required and usually represented as a direction field or orientation field (OF) [5]. The pattern around a singularity has its unique structure and can be prescribed as flow template [6]. These special structures are used to allocate type of singularity and its location [7]. There is a huge number of well-documented methods used to identify singularities of fingerprint images [8], especially the Poincare index method [7]. Major methods work well for high quality images; however, when the images include high level of noises or irregular structures, spurious points are obtained [9]. Several methods such as phase portrait analysis [10] or Poincare index are suffered from fixed size of searching window; consequently, the singularities near boundary image are unable to detect [11]. Moment invariants [12] are properties of connected regions in binary images that are invariant to translation, rotation and scale. The use of moment invariants to determine the singularity of a fingerprint image has not been well investigated and applied, especially for development of algorithms to detect the singularity of fingerprint images.

The rest of the paper is organized as follows. Section 2 presents the procedure to extract the orientation field (OF) of fingerprint images. The moment invariant formulation for the resulted OF is presented in Section 3. The proposed fingerprint singularity detection algorithm is presented in Section 4. Finally, Section 5 presents the test results, conclusions and brief discussions.

\section{Estimation of the orientation fields}

The flow patterns established by the ridge and valley of fingerprint images are represented by bi-direction vector fields (VFs) or orientation fields (OFs). Usually, the ridge orientation ranges from 0 to $\pi$. Conveniently, the range of orientation is defined in [$\pi / 2, \pi / 2]$. A number of methods have been proposed to extract the OF from the fingerprint images [5]. A robust and efficient method to noise images proposed in [13] is used to extract the OFs of fingerprint images in this study. For a given normalized image $I(x$, $y$ ), five steps (S1 to S5) to evaluate the orientation at pixel $(x, y)$ are presented as follows: (S1) A block size $W \times W$ is centered at $(x, y)$; (S2) For each pixel in the block, compute the gradient $\partial_{x} I(x, y)$ and $\partial_{y} I(x, y)$; (S3) The local orientation $\theta(x, y)$ then 
can be estimated using the equations: $\theta(x, y)=\frac{1}{2} \tan ^{-1} \frac{V_{y}(x, y)}{V_{x}(x, y)} \quad$ where $V_{x}(x, y)=\sum_{u=x-\frac{W}{2}}^{x+\frac{W}{2}} \sum_{v=y-\frac{W}{2}}^{y+\frac{W}{2}} 2 \partial_{x} I(u, v) \partial_{y} I(u, v)$ and $V_{y}(x, y)=\sum_{u=x-\frac{W}{2}}^{x+\frac{W}{2}} \sum_{v=y-\frac{W}{2}}^{y+\frac{W}{2}} \partial_{x}^{2} I(u, v) \partial_{y}^{2} I(u, v)$;

(S4) Smooth the OF in a local neighborhood using the Gaussian filter to correct the corrupted orientation which is resulted from noises; and (S5) Finally, the orientation at the pixel $(x, y)$ now can be re-assigned as: $\theta(x, y)=\frac{1}{2} \tan ^{-1} \frac{U_{x}(x, y)}{U_{y}(x, y)}$, where $U_{x}(x, y)$ and $U_{y}(x, y)$ are the components of the continuous vector field with the Gaussian filter. Examples of fingerprint orientation extractions and estimations are presented in Fig.1.
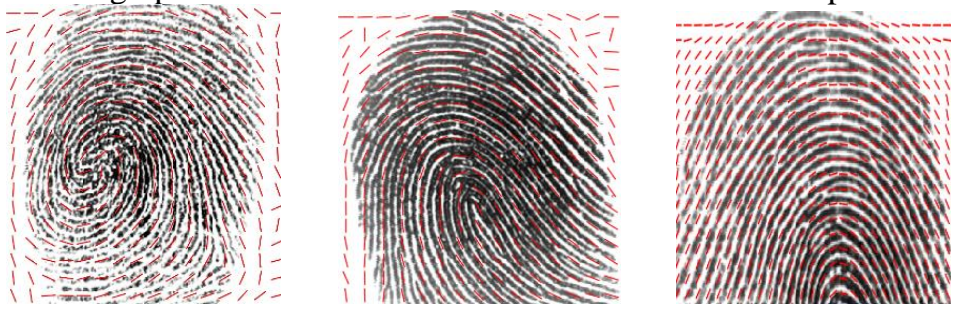

Fig. 1. Fingerprint orientation field extraction

\section{Formulation of the moment invariant}

Let us represent the orientation of the ridge at the location $(x, y)$ by a unit vector $\left(v_{x}, v_{y}\right)$ and express it in form of a complex number $V=v_{x}+i v_{y}$ defined in the reference coordinate system. The "argument" of the complex number is defined by $\theta(x, y)=\left\{\begin{array}{l}\tan ^{-1}\left(v_{y} / v_{x}\right), \text { if } v_{x} \neq 0, \quad \text { Clearly, } \quad \theta(x, y)=\theta(-x,-y) \quad \text { and } \\ -\pi / 2, \text { otherwise. }\end{array}\right.$ $\theta(-x, y)=\theta(x,-y)$. Therefore, $f(x, y)=e^{i \theta(x, y)}$ is "flip invariant" and can represent the OF. A complex moment $c_{p q}$ is defined by [12]: $c_{p q}=\int_{-\infty}^{\infty} \int_{-\infty}^{\infty}(x+i y)^{p}(x-i y)^{q} f(x, y) d x d y$. We replace the integral $\int_{-\infty}^{\infty} \int_{-\infty}^{\infty}$ by an integral over a sampling circle with the radius $R_{s}$. In the polar coordinates $c_{p q}=\int_{0}^{R_{s}} \int_{0}^{2 \pi} r^{p+q+1} e^{i(p-q) \varphi} f(r, \varphi) d r d \varphi . \quad$ Substituting $\quad f(r, \varphi)=e^{i \theta(r, \varphi)} \quad$ yields $c_{p q}=\int_{0}^{R_{s}} \int_{0}^{2 \pi} r^{p+q+1} e^{i(p \varphi-q \varphi+\theta(r, \varphi))} d r d \varphi$. Note that $0 \leq \varphi \leq 2 \pi$, whereas $-\frac{\pi}{2} \leq \theta \leq \frac{\pi}{2}$. If $f(r, \varphi)=e^{i \theta(r, \varphi)}$ is rotated by an angle $\alpha, \quad f_{\text {new }}(r, \varphi)=e^{i(\theta(r, \varphi)-\alpha)}$, then 
$c_{p q, \text { new }}=e^{i(p-q+1) \alpha} c_{p q}$. Therefore, any moment constructed as $\prod_{j=1}^{n} c_{p_{j} q_{j}}$ with $\sum_{j=1}^{n}\left(p_{j}-q_{j}+1\right)=0 \quad$ is rotationally invariant. A change of variables $\hat{x}=x-\bar{x}, \hat{y}=y-\bar{y}$ and normalizing $c_{p q}$ implies the translation \& scale invariance as follows: $c_{p q}=\frac{1}{v^{\gamma}} \int_{0}^{\infty} \int_{0}^{\infty}(\hat{x}+i \hat{y})^{p}(\hat{x}-i \hat{y})^{q} f(\hat{x}, \hat{y}) d \hat{x} d \hat{y}$, where $\gamma=\frac{p+q+2}{2}, v=\int_{-\infty}^{+\infty} \int_{-\infty}^{+\infty} \chi(\hat{x}, \hat{y}) d \hat{x} d \hat{y}$, $\chi(x, y)=\left\{\begin{array}{l}1, \text { if }(x, y) \in W_{s} \\ 0, \text { otherwise, }\end{array}, \bar{x}=\frac{\int_{-\infty}^{+\infty} \int_{-\infty}^{+\infty} x \chi(x, y) d x d y}{\int_{-\infty}^{+\infty} \int_{-\infty}^{+\infty} \chi(x, y) d x d y}, \bar{y}=\frac{\int_{-\infty}^{+\infty} \int_{-\infty}^{+\infty} y \chi(x, y) d x d y}{\int_{-\infty}^{+\infty} \int_{-\infty}^{+\infty} \chi(x, y) d x d y}\right.$.

Finally, Flusser [12] derives a set of independent moments of the order $p+q \leq 2$ as follows: $\mu_{2}=\left\{\mathrm{c}_{01}, \mathrm{c}_{00} \mathrm{c}_{02}, \mathrm{c}_{11} \mathrm{c}_{02}, \mathrm{c}_{10} \mathrm{c}_{02}^{2}, \mathrm{c}_{20} \mathrm{c}_{02}^{3}\right\}$. The moments are considered as a feature vector in a multi-dimensional space. In order to identify the patterns a straightforward test is applied to compare a vector $\mu_{2}$ with a template vector $\mu_{2, \text { pattern }}$ and an identification threshold $\zeta$ is introduced $[14,15]$.

\section{Algorithm for detection of singularity of fingerprint images}

The proposed algorithm is presented as follows.

1. Generation of a singularity template. From a set of fingerprint images with well defined singularities, a sample of patterns centered at the singularity pixels with different radiuses is selected for each type of singularity. Moment invariant vectors are evaluated, averaged, and stored as template vector $\mu_{2, \text { pattern }}$.

2. Calculation of the pyramid moment invariant. For an input fingerprint image of size $M \times N$. Define a moving square window centered at $(x, y)$ with the radius $k$. For each position $(x, y)$, a sequence of moments $B_{x, y}^{k}$ is evaluated. The increasing radius of the window $k=k_{\min }, \ldots, k_{\max }$ creates a pyramid structure [16].

3. Calculation of the similarity pyramid. For each type of singularity, a same structure of pyramid moment is constructed by replacing the value in each cell by the Euclidean distance between the vector in the cell and the template vector.

4. Detection of singularities. The pyramid moment is expressed in a matrix form and sorted in descending order of similarity (higher similarity means lower the distance, thus closer matching pattern). An $\varepsilon_{1}-$ threshold cut-off level is performed. A new set of $\varepsilon_{1}$ - similarity with different window sizes is obtained. Now, the obtained set is sorted by descending the order of a window radius. The similarity value corresponding to the smallest window size will again be used as the minimum similarity threshold, $\varepsilon_{2}$. Remove any rows that have a similarity smaller than $\varepsilon_{2}$. The process is repeated until the singularities are found. 


\section{$5 \quad$ Results, discussions and conclusions}

The proposed fingerprint singularity detection algorithm was successfully developed and it was then verified by using two common sources of data bases FVC2002/DB1 and FVC2002/DB2 [17]. The data DB1 is used for the singularity template extraction. The searching window radius is selected from 10 to 50 with the increment size 10 . The proposed algorithm and the Poincare index method were then tested using both databases. The experiment results are shown in Table 1. Note that the size of the OFs can be defined for appropriate image resolutions and to reduce the computational cost.

Table 1. Experiment results: A singularity detection rate.

\begin{tabular}{lcccc}
\hline & \multicolumn{2}{c}{ DB1 } & \multicolumn{2}{c}{ DB2 } \\
\cline { 2 - 5 } & Core & Delta & Core & Delta \\
\hline Actual value & 80 & 34 & 79 & 60 \\
Poincare Index [7] & 80 & 27 & 73 & 47 \\
Moment Invariant & 80 & 33 & 76 & 55 \\
PI - detection rate (\%) & 100 & 79.4 & 92.4 & 78.3 \\
MI- detection rate (\%) & 100 & 97.0 & 96.2 & 91.6 \\
\hline
\end{tabular}

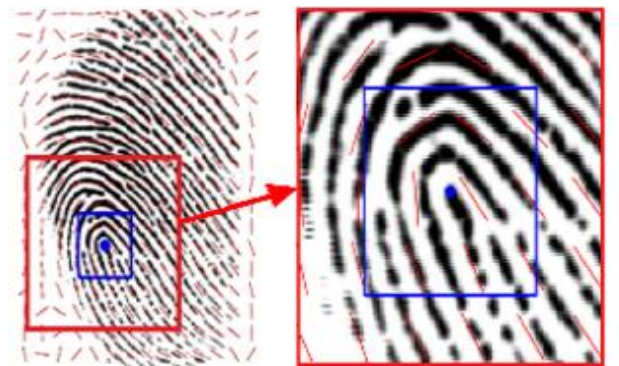

(a)

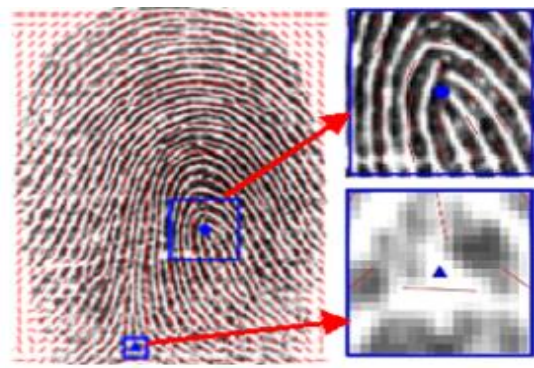

(b)

Fig. 2. (a): Core and delta detection. (b): Detection of a singularity closed to the image boundary.

Figure 2 presents the core and delta detection examples. The obtained results show that the moment invariant algorithm in this study outperforms the Poincare index and it is able to detect the singularity that is very closed to the image boundary (Fig. 2b).

In conclusion, the fingerprint singularity detection algorithm was successfully developed and tested for different fingerprint image resolutions and noise levels. The proposed algorithm is robust and can be adopted to any special descriptors rather than the pure core and delta, with potentials for biometrics and medical applications. The outcome of a study is directly used for further development of a complete fingerprint authentication system to safeguard privacy and grant access to EHRs, aimed to enhance the security as well as effective managements and use of patient data and medical records in telehealth, management of confidential medical records, and e-health systems. 
Disclosure statement. No conflict of interest was reported by the authors.

Acknowledgement. This research is sponsored by the Center of Excellency in Biomedical Engineering, Thammasat University of Thailand, British Council and Newton Fund.

\section{References}

1. Chen, Y-C., Chen, L-K., Tsai, M-D., Chiu, H-C., Chiu, J-S., Chong, C-F.: Fingerprint verification on medical image reporting system. Comput Methods and Programs in Biomed 89 (3):282-288(2008).

2. Sadhya, D., Singh, S. K.: Privacy risks ensuing from cross-matching among databases: A case study for soft biometrics. Inform Process Lett 128:38-45(2017).

3. Yang, W., Wang, S., Hu, J., Zheng, G., Valli, C.: A fingerprint and finger-vein based cancelable multi-biometric system. Pattern Recogn 78:242-251(2018).

4. Weerasinghe, D., Rahulamathavan, Y., Rajarajan, M: Secure trust delegation for sharing patient medical records in a mobile environment. Health Policy and Technology 2:3644(2013).

5. Gupta, P., Gupta, P.: An accurate fingerprint orientation modeling algorithm. Applied Mathematical Modelling 40:7182-7194(2016).

6. Dass, S. C., Jain, A. K.: Markov models for directional field and singularity extraction in fingerprint images. IEEE Transaction Image Processing 13(10):1358-1367(2004).

7. Kawagoe, M., Tojo, A.: Fingerprint pattern classification. Pattern Recognition 17(3):295303(1984).

8. Iwasokun, G. B., Akinyokun, O. C.: Fingerprint singular point detection based on modified Poincare index method. Int J Sig Process, Image Prcess and Pat Recog 7(5):259-272(2014).

9. Zacharias, G. C., Lal, P. S.: Singularity detection in fingerprint image using orientation consistency. 2013 International Mutli-Conference on Automation, Computing, Communication, Control and Compressed Sensing (iMac4s), pp.150-154 (2013).

10. Ram, S., Bischof, H., Birchbauer, J.: Detection fingerprint singularity using linear phase portraits. In: Handbook of Remote Biometrics. Advances in Pattern Recognition, pp. 349-362. Springer, London (2009).

11. Li, J., Yau, W-Y., Wang, H.: Combining singular points and orientation image information for fingerprint classification. Pattern Recognition 41:353-366(2008).

12. Flusser, J., Suk, T., Zitová, B.: Moments and moment invariants in pattern recognition. Wiley, Chichester (2009).

13. Thai, R.: Fingerprint image enhancement and minutiae extraction. The Univ. Western Australia (2003).

14. Flusser, J., Suk, T.: Affine moment invariants: a new tool for character recognition. Pattern Recognit Lett 15:433-436(1994).

15. Hu, M-K.: Visual pattern recognition by moment invariants. IRE Trans Inf Theory 8:179187(1962).

16. Schlemmer, M., Heringer, M., Morr, F., Hotz. I., Bertram, MH., Garth, C., Kollmann, W., Hamann, B., Hagen, H.: Moment invariants for the analysis of 2D flowfields. Visualization and Comput Graphics IEEE Transactions 13: 1743-1750(2007).

17. Maio, D., Maltoni, D., Cappelli, R., Wayman, J. L., Jain A. K.: "FVC2002: Fingerprint verification competition", Proc. Int. Conf. Pattern Recognition (ICPR), pp.744-747 (2002). 